\title{
Plasma level of cyclophilin A is increased in patients with type 2 diabetes mellitus and suggests presence of vascular disease
}

Surya Ramachandran ${ }^{1}$, Anila Venugopal ${ }^{1}$, V Raman Kutty ${ }^{2}$, Vinitha A ${ }^{1}$, Divya $G^{1}$, V Chitrasree $^{3}$, Ajit Mullassari ${ }^{3}$, N S Pratapchandran ${ }^{4}$, K R Santosh ${ }^{5}$, M Radhakrishna Pillai ${ }^{6}$ and C C Kartha ${ }^{{ }^{*}}$

\begin{abstract}
Aims/hypothesis: Cyclophilin A, an immunophilin is secreted from human monocytes activated by high glucose. Given its role as an inflammatory mediator of vascular tissue damage associated with inflammation and oxidative stress, we examined plasma levels of cyclophilin A in normal healthy volunteers and patients with type 2 diabetes (DM), with or without coronary artery disease (CAD).

Methods: Study subjects comprised of 212 patients with DM and CAD,101 patients with diabetes, 122 patients with CAD and 121 normal healthy volunteers. Diabetes was assessed by HbA1c levels while coronary artery disease was established by a positive treadmill test and/or coronary angiography. Plasma cyclophilin A was measured using a cyclophilin A ELISA Kit. Relationship of plasma cyclophilin A levels with blood markers of type 2 diabetes, blood lipid levels and medication for diabetes and coronary artery disease were also explored.

Results: Plasma Cyclophilin levels were higher in diabetes patients with or without CAD compared to normal subjects $(\mathrm{P}<0.001)$. Age, fasting blood sugar levels and $\mathrm{HbA1C}$ levels were positively associated with increased plasma cyclophilin. Patients using metformin had reduced levels of plasma cyclophilin $(p<0.001)$.Serum levels of total cholesterol, LDL cholesterol and triglycerides had no significant association with plasma cyclophilin levels. In patients with increased serum CRP levels, plasma cyclophilin A was also elevated ( $p=0.016)$. Prevalence odds for $D M, D M+C A D$ and CAD are higher in those with high cyclophilin values, compared to those with lower values, after adjusting for age and sex, indicating strong association of high cyclophilin values with diabetes and vascular disease.
\end{abstract}

Conclusions/interpretations: Our study demonstrates that patients with type 2 diabetes have higher circulating levels of cyclophilin A than the normal population. Plasma cyclophilin levels were increased in patients with diabetes and coronary artery disease suggesting a role of this protein in accelerating vascular disease in type 2 diabetes. Considering the evidence that Cyclophilin A is an inflammatory mediator in atherogenesis, the mechanistic role of cyclophilin A in diabetic vascular disease progression deserves detailed investigation.

Keywords: Hyperglycemia, Type 2 diabetes mellitus, Monocytes, Cyclophilin A, Vascular disease

\footnotetext{
* Correspondence: cckartha@rgcb.res.in

'Cardiovascular Disease Biology, Rajiv Gandhi Centre for Biotechnology,

Thiruvananthapuram, India

Full list of author information is available at the end of the article
} 


\section{Introduction}

Cyclophilin A is part of various intracellular functions, such as intracellular signaling, protein trafficking, and regulating activity of other proteins [1]. Cyclophilin A, is also well recognized as a secreted growth factor that is induced by oxidative stress [2] functioning as a mediator of tissue damage associated with inflammation and oxidative stress [3]. The secretory nature of this protein and its presence in plasma of patients with DM and CAD underlines its potential as a marker of disease. We earlier reported proteomic changes occurring in circulating monocytes on activation with high glucose. We also demonstrated secretion of cyclophilin A from monocytes under hyperglycemic conditions. Preliminary studies on a small number of patients with type 2 diabetes mellitus and coronary artery disease revealed increased plasma levels of cyclophilin A compared to normal healthy individuals [4].

We report here the results of an enzyme linked immunosorbent assay based study in a larger study population comprising 556 subjects, consisting of patients with type 2 diabetes mellitus with or without coronary artery disease, patients with only coronary artery disease and normal healthy volunteers. Levels of plasma cyclophilin A in these study groups was determined and correlated with biochemical markers of diabetes and blood lipid profile.

\section{Methods}

\section{Study participants}

The study was done on patients with type 2 diabetes with or without macrovascular complications such as coronary artery disease. Patients were recruited from three multispecialty hospital centers in Southern India. All the protocols were submitted to and approved by the Human Ethics Committee of Rajiv Gandhi Centre for Biotechnology and participating hospitals (Indian Institute of Diabetes, PRS Hospital and Madras Medical Mission). A written informed consent was obtained from all study subjects. All patients subsequently underwent a standardized clinical and laboratory evaluation.

The study subjects $(\mathrm{n}=556)$ were divided into five groups: (i) Patients with type 2 diabetes mellitus (DM; $\mathrm{n}=$ 101), (ii) diabetes patients with coronary artery disease $(\mathrm{DM}+\mathrm{CAD})$ diagnosed within 5 years of detection of type 2 diabetes $(\mathrm{n}=103)$, (iii) patients with DM who had CAD diagnosed five years after detection of diabetes $(n=109)$, (iv) patients with CAD $(\mathrm{n}=122)$ and without diabetes and (v) normal healthy volunteers $(\mathrm{n}=121)$.

Patients with hypertension, cardiac diseases other than coronary artery disease, peripheral vascular disease, thrombotic stroke, nephropathy, retinopathy, inflammatory disease of any cause, subjects with other systemic and metabolic diseases, asthma, malignancy, liver diseases, kidney diseases and pregnant women were excluded from the study. Diabetes was assessed by recording HbA1c and/or fasting blood sugar (FBS) levels while CAD was diagnosed by a positive treadmill test and/or coronary angiography.

\section{Enzyme linked assay for measurement of cyclophilin A in plasma}

Blood samples were collected after overnight fasting. Blood was collected into EDTA containing tubes and centrifuged for 7 minutes at $2500 \mathrm{~g}$ within 30 minutes of collection. The separated plasma was transferred to a fresh tube and used directly for the immunoassay as per the supplier's protocol. Cyclophilin A levels in plasma were determined with a sandwich immunoassay kit (Uscn Life Science Inc, Product No. SEA979Hu). The linearity of the kit was assayed by testing samples spiked with a known concentration of cyclophilin A and their serial dilutions. Spiking with known concentrations of the protein guaranteed a recovery range of $83-102 \%$. No significant cross-reactivity or interference with any other proteins was observed. All samples were analyzed in duplicate. To maintain assay precision, samples with a $\mathrm{CV}>12 \%$ were excluded.

C-Reactive Protein (CRP) was measured with a quantikine sandwich Human CRP Immunoassay (R \& D Systems, Product No: DCRP00). A 93-100\% recovery range was observed when samples were spiked with 1:2 to $1: 16$ concentrations of CRP.

Medication history was recorded for 270 subjects of the 556 subjects. Medications were grouped into (i) antiplatelet aggregating agents (Ecospirin, Clopidogrel, Cilostazol), (ii) Antihypertensive agents (Calcium channel blockers, ACE inhibitors, vasodilators), (iii) Statins (atorvastatin, Lovastatin) and (iv) Metformin derivatives (glucophage, glycimet).

\section{Statistical analysis}

The distribution of all variables in the five groups was studied by describing the mean, median, range and standard deviation. Predictor variables such as age, HbA1C, fasting blood sugar (FBS) were grouped into two based on median values; cyclophilin A levels in the two groups compared using student $t$ test. The same procedure was used for comparing cyclophilin levels in groups with different levels of CRP, and in patients using metformin or not. Cyclophilin values in the five groups were compared with ANOVA $(\mathrm{F}=54.75, \mathrm{p}<0.001)$, followed by multiple comparisons using pairwise $t$ tests with pooled variance by the Holms' method. We did a multinomial logistic regression analysis for estimating the prevalence odds ratios for presence of disease, with the normal subjects as the reference. The odds for prevalence of the other four conditions, i.e., diabetes and no heart disease (DM), heart 
disease and no diabetes (CAD), diabetes and heart disease of up to 5 years duration (DM + CAD 5Y), and diabetes and heart disease beyond 5 years but below 10 years $(\mathrm{DM}+\mathrm{CAD} 10 \mathrm{Y})$, among those subjects with high cyclophilin levels were compared with prevalence odds in those with low cyclophilin values, adjusted for age and sex. All statistical tests were done with an estimated power $=0.80$. All statistical tests were done in $\mathrm{R}$ (R Core Team , 2013, R: A language and environment for statistical computing. R Foundation for Statistical Computing, Vienna, Austria.); graphs were also created in $\mathrm{R}$.

\section{Results}

Baseline clinical parameters of patients with diabetes, coronary artery disease and controls

A total of 313 diabetes patients; 212 with CAD and 101 without CAD (210 men and 103 women), 122 patients with only coronary artery disease (102 men and 20 women) and 121 normal healthy volunteers (66 men and 55 women) without any evidence of systemic disease participated in the study. The distribution of study subjects and their baseline clinical characteristics are described in Table 1. The mean values and standard deviation of each clinical parameter is given in Table 2 . Mean HbA1c values were highest in the diabetes mellitus (DM) group (mean: 8.3; Std dev: 1.3) followed by the group of patients with diabetes and coronary artery disease (DM + CAD10y) (mean: 8.6, Std dev: 2.0). Triglyceride levels were higher in patients with coronary artery disease irrespective of whether they had diabetes compared to normal subjects and patients with only diabetes.

\section{Levels of plasma cyclophilin A and association with disease}

Plasma cyclophilin in the 556 subjects ranged from $5.9 \mathrm{ng} / \mathrm{ml}$ to $59.2 \mathrm{ng} / \mathrm{ml}$ ) with a median value of 16.7 $\mathrm{ng} / \mathrm{ml}$. Subjects were divided into two groups: subjects with plasma cyclophilin $<16.7 \mathrm{ng} / \mathrm{ml}$ and those above

Table 1 Frequencies of baseline clinical parameters of the study groups

\begin{tabular}{|c|c|c|c|c|c|}
\hline & Normal N (\%) & DM N (\%) & CAD N (\%) & $\mathrm{DM}+\mathrm{CAD}(0-5 \mathrm{y}) \mathrm{N}(\%)$ & $\mathrm{DM}+\mathrm{CAD}(5-10 \mathrm{y}) \mathrm{N}(\%)$ \\
\hline \multicolumn{6}{|l|}{ Age } \\
\hline$>45$ & 47 (38.8) & $18(18)$ & $13(10.6)$ & $6(5.9)$ & $3(2.8)$ \\
\hline$<45$ & $74(61.1)$ & $83(83)$ & 109 (85.3) & $97(95.1)$ & $106(97.2)$ \\
\hline \multicolumn{6}{|l|}{ Gender } \\
\hline Male & $66(54.5)$ & $49(49)$ & $102(83.6)$ & $83(81.4)$ & 78 (71.6) \\
\hline Female & $55(48.4)$ & $52(52)$ & $20(16.3)$ & $20(19.6)$ & $3(28.4)$ \\
\hline \multicolumn{6}{|l|}{ FBS (mg/dL) } \\
\hline $70-110$ & $89(73.5)$ & $26(26)$ & $43(35.2)$ & $25(24.5)$ & $21(19.3)$ \\
\hline$>110$ & $32(26.4)$ & $75(75)$ & $79(64.7)$ & $78(76.5)$ & $88(80.7)$ \\
\hline \multicolumn{6}{|l|}{ HbA1C (\%) } \\
\hline$<5.7$ & $88(72.7)$ & $4(4)$ & $50(40.9)$ & $1(1)$ & $3(2.8)$ \\
\hline $5.7-6.4$ & $28(23.1)$ & $6(6)$ & $40(32.70$ & 19 (18.6) & $5(4.6)$ \\
\hline$>6.5$ & $5(4.1)$ & $91(91)$ & $32(26.2)$ & $83(81.4)$ & $101(92.7)$ \\
\hline \multicolumn{6}{|c|}{ Total cholesterol (mg/dL) } \\
\hline$<200$ & $64(52.8)$ & $58(58)$ & $90(73.7)$ & $78(76.5)$ & $80(73.4)$ \\
\hline $200-240$ & $37(30.5)$ & $36(36)$ & $15(72.2)$ & $12(11.8)$ & $13(11.9)$ \\
\hline$>240$ & $20(16.5)$ & $7(7)$ & $17((13.9)$ & $13(12.7)$ & $16(14.7)$ \\
\hline \multicolumn{6}{|l|}{ LDL (mg/dL) } \\
\hline$<100$ & $91(75.2)$ & $33(33)$ & $78(63.9)$ & $68(66.7)$ & $64(58.7)$ \\
\hline$>100$ & $30(24.7)$ & $68(68)$ & $44(36.1)$ & $35(34.3)$ & $45(41.3)$ \\
\hline \multicolumn{6}{|c|}{$\mathrm{HDL}(\mathrm{mg} / \mathrm{dL})$} \\
\hline$<60$ & $113(43.3)$ & $90(90)$ & $117(95.9)$ & $100(98)$ & $104(95.4)$ \\
\hline$>60$ & $8(6.6)$ & $11(11)$ & $5(4.1)$ & $3(2.9)$ & $5(4.6)$ \\
\hline \multicolumn{6}{|c|}{ Triglycerides (mg/dL) } \\
\hline$<150$ & $113(43.3)$ & $90(90)$ & $78(63.9)$ & $64(62.7)$ & 77 (70.6) \\
\hline$>150$ & $8(6.6)$ & $11(11)$ & $44(36.1)$ & $39(38.2)$ & $32(29.4)$ \\
\hline
\end{tabular}


Table 2 Mean values of clinical parameters in the study groups

\begin{tabular}{|c|c|c|c|c|c|c|c|c|c|c|}
\hline & \multicolumn{2}{|c|}{$\mathrm{N}$} & \multicolumn{2}{|c|}{$\mathrm{DM}$} & \multicolumn{2}{|c|}{ CAD } & \multicolumn{2}{|c|}{$\mathrm{DM}+\mathrm{CAD} 5 \mathrm{y}$} & \multicolumn{2}{|c|}{$\mathrm{DM}+\mathrm{CAD} 10 \mathrm{y}$} \\
\hline & $M$ & SD & $M$ & SD & $M$ & SD & $M$ & SD & $M$ & SD \\
\hline Age & 41.6 & 11.2 & 55.2 & 10.0 & 58.9 & 11.2 & 58.8 & 9.0 & 62.4 & 8.4 \\
\hline FBS & 106.6 & 25.8 & 149.8 & 54.6 & 124.8 & 31.4 & 145.6 & 52.8 & 162 & 58.1 \\
\hline $\mathrm{HbA1c}$ & 5.3 & 0.5 & 8.3 & 1.3 & 6.0 & 1.1 & 7.7 & 1.6 & 8.6 & 2.0 \\
\hline Total cholesterol & 199 & 42 & 192.9 & 34.5 & 167.7 & 56.2 & 172.1 & 60.6 & 170.5 & 56.0 \\
\hline LDL & 92.1 & 23.9 & 110.6 & 22.5 & 102 & 36.6 & 96.9 & 40.2 & 105.5 & 39.3 \\
\hline HDL & 43.5 & 8.5 & 47.4 & 9.2 & 42.4 & 12.0 & 36.1 & 10.1 & 38.6 & 11.7 \\
\hline Triglycerides & 107.4 & 29.6 & 103.2 & 33.2 & 136.6 & 49.0 & 152.9 & 81.5 & 134.4 & 58.4 \\
\hline Cyclophilin A & 13.2 & 3.8 & 16.1 & 4.0 & 20.0 & 8.0 & 18.7 & 9.4 & 19.3 & 8.2 \\
\hline
\end{tabular}

Mean values of clinical parameters in the study groups. $N=$ Normal, $D M=$ Diabetes mellitus, $C A D=$ Coronary artery disease, $D M+C A D 5 y=$ diabetes patients with coronary artery disease $(D M+C A D)$ diagnosed within 5 years of detection of type 2 diabetes, DM+CAD10y = patients with DM who had CAD diagnosed five years after detection of diabetes; $M=$ Median values and $S D=$ Standard deviation.

$16.7 \mathrm{ng} / \mathrm{ml}$. There was no significant difference in plasma cyclophilin levels between males and females $(\mathrm{p}=0.72)$. We observed that only age $(\mathrm{t}=3.93 ; \mathrm{p}<0.01)$, FBS $(\mathrm{t}=$ 6.19; $\mathrm{p}<0.01)$ and $\mathrm{HbA1C}(\mathrm{t}=2.34 ; \mathrm{p}=0.019)$ were associated with changes in plasma cyclophilin A levels (Figure 1). Other variables such as serum levels of total cholesterol, LDL cholesterol and triglycerides had no significant association with plasma cyclophilin levels. Figure 2 describes the distribution of cyclophilin levels among the various study groups. The plasma levels of cyclophilin A were significantly higher in patients with type 2 diabetes (DM) in comparison to normal volunteers $(\mathrm{p}<0.001)$. Plasma levels were of normal distribution in the control group whereas in other groups the distribution was skewed. We used ANOVA to look into the association of cyclophilin A levels in 5 groups of subjects: (i) patients with diabetes (DM) and diagnosed to have CAD within 5 years of onset of diabetes (DM + CAD5y), (ii) patients with DM and diagnosed to have CAD after 5 years and within 10 years of onset of diabetes (DM + CAD 10y), (iii) patients with only diabetes, (iv) patients with only diabetes, and (v) controls. The analysis revealed that the normal group $(\mathrm{N})$ was different from all other groups, and the diabetic group (DM) was different from all other groups. The CAD group did not differ from the DM + CAD groups (both DM + CAD5y and DM + CAD10y) with respect to cyclophilin levels, but were distinctly different from normal $(\mathrm{N})$ as well as diabetes patient (DM) group.

Multinomial logistic regression analysis revealed that prevalence odds for all four conditions (DM, DM + CAD $5 Y, D M+C A D 10 y$ and CAD) are higher in those with high cyclophilin values, compared to those with lower values, after adjusting for age and sex, indicating strong association of high cyclophilin values with diabetes and
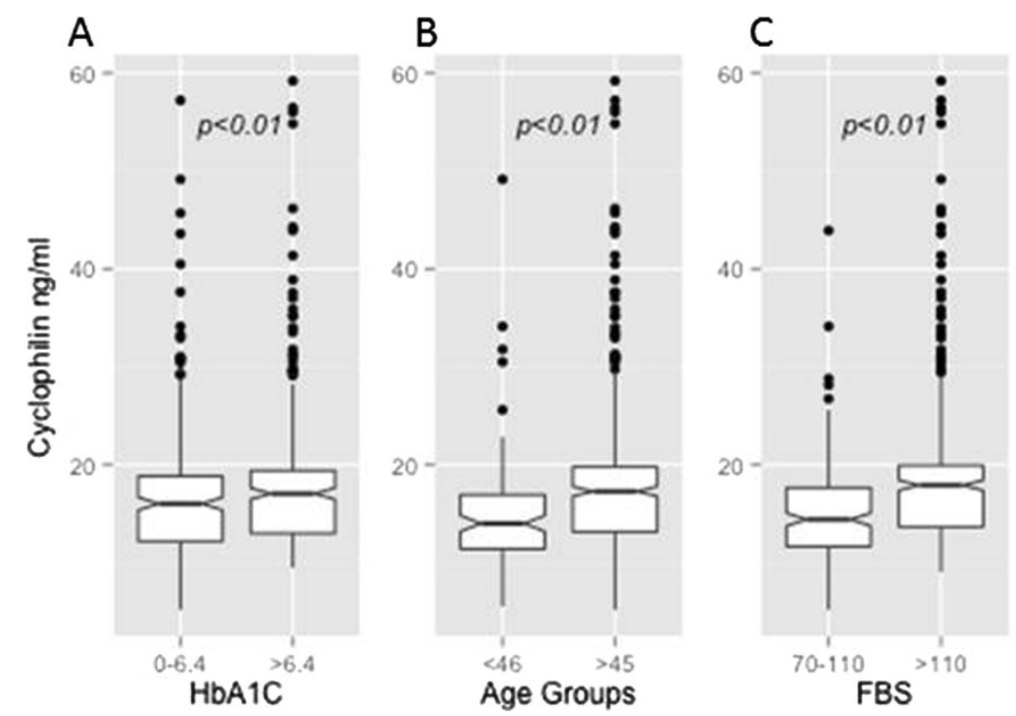

Figure 1 Box and whisker plot of association between (A) HbA1C (B) Age and (C) Fasting blood sugar (FBS) and plasma cyclophilin levels. HbAlc was divided into $<6.4$ and 6.4 and above age was divided into $<46$ and $>45$ and FBS between $70-110 \mathrm{mg} / \mathrm{dL}$ and $>110 \mathrm{mg} / \mathrm{dL}$. 


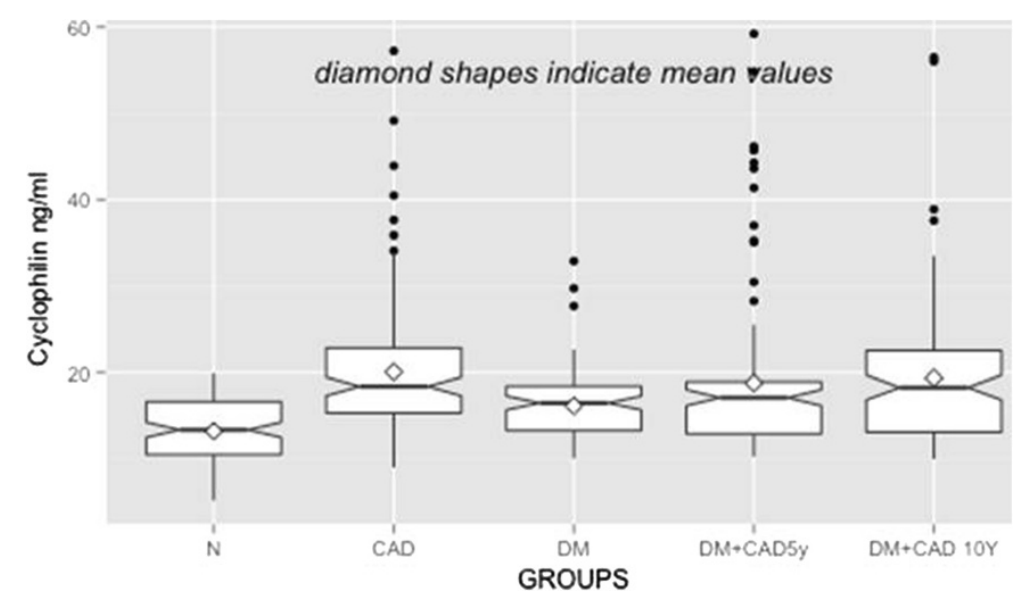

Figure 2 Box and whisker plot of distribution of cyclophilin levels in plasma of the various study groups. $\mathrm{Nt}=\mathrm{Normal}$ control; $\mathrm{CAD}=\mathrm{Coronary}$ artery disease; $\mathrm{DM}=$ Diabetes Mellitus, DM+CAD5y = diabetes patient diagnosed with CAD in 5 years and DM+CAD1Oy = diabetes patients diagnosed with CAD within 10 years. P values were $<0.001$ for all groups.

vascular disease. However, the prevalence odds of those with both diabetes and heart disease were lower compared to those subjects who had only heart disease; the last mentioned group had the strongest association with high cyclophilin values. The prevalence odds ratios and 95\% confidence interval are given in Table 3.

Association of $C$ reactive protein with cyclophilin $A$ levels It is well recognized that patients with low serum CRP $(<1 \mathrm{mg} / \mathrm{L})$ are at low risk and those with higher CRP $(>1 \mathrm{mg} / \mathrm{L})$ are at moderate or high risk for future cardiovascular events [5]. We evaluated the plasma levels of cyclophilin A among patients with high and low levels of CRP. Plasma cyclophilin A levels in patients with higher serum CRP $(>1.4 \mathrm{mg} / \mathrm{L}, \mathrm{n}=38)$ and lower serum CRP $(<1.4 \mathrm{mg} / \mathrm{L}, \mathrm{n}=39)$ are shown as box-and-whisker plots in Figure 3. There was a positive association between higher serum CRP levels and higher plasma cyclophilin A levels $(P=0.016)$ compared with plasma cyclophilin levels in the low serum CRP group.

Table 3 Prevalence Odds Ratios (POR) and 95\% confidence intervals for association of disease conditions with high vs low cyclophilin levels, adjusted for age and sex, using multinomial logistic regression analysis

\begin{tabular}{lcc}
\hline Groups* & Prevalence odds ratio & $\mathbf{9 5 \%}$ Cl on POR \\
\hline DM $(\mathbf{n}=\mathbf{1 0 1})$ & 2.37 & $1.26-4.44$ \\
DM+CAD 5Y $(\mathbf{n}=\mathbf{1 0 3})$ & 2.99 & $1.56-5.71$ \\
DM+CAD 10Y $(\mathbf{n}=\mathbf{1 0 9})$ & 3.76 & $1.97-7.17$ \\
CAD $(\mathbf{n}=\mathbf{1 2 2})$ & 5.17 & $2.76-9.71$
\end{tabular}

*Normal subjects were the referent group. DM = subjects with diabetes only; $\mathrm{DM}+\mathrm{CAD} 5 \mathrm{Y}=$ subjects with diabetes and coronary disease of $<5$ years' duration; $\mathrm{DM}+10 \mathrm{Y}=$ subjects with diabetes and coronary disease with duration $<10$ years but $>5$ years.

\section{Cyclophilin levels and medication}

Cyclophilin levels were positively associated with use of metformin (Table 4). The plasma levels of cyclophilin were low in those regularly taking metformin $(\mathrm{p}<0.001)$. None of the other medications were found to be associated with plasma cyclophilin A levels.

\section{Discussion}

We describe here the difference in circulating levels of plasma Cyclophilin A in patients with diabetes in comparison to normal individuals without any systemic disease. We used an ELISA method to quantify the cyclophilin A levels in human plasma. The method is accurate and specific for detection of the immunophilin in plasma. Cyclophilin A was considerably high in the plasma of patients with type 2 diabetes. The levels were

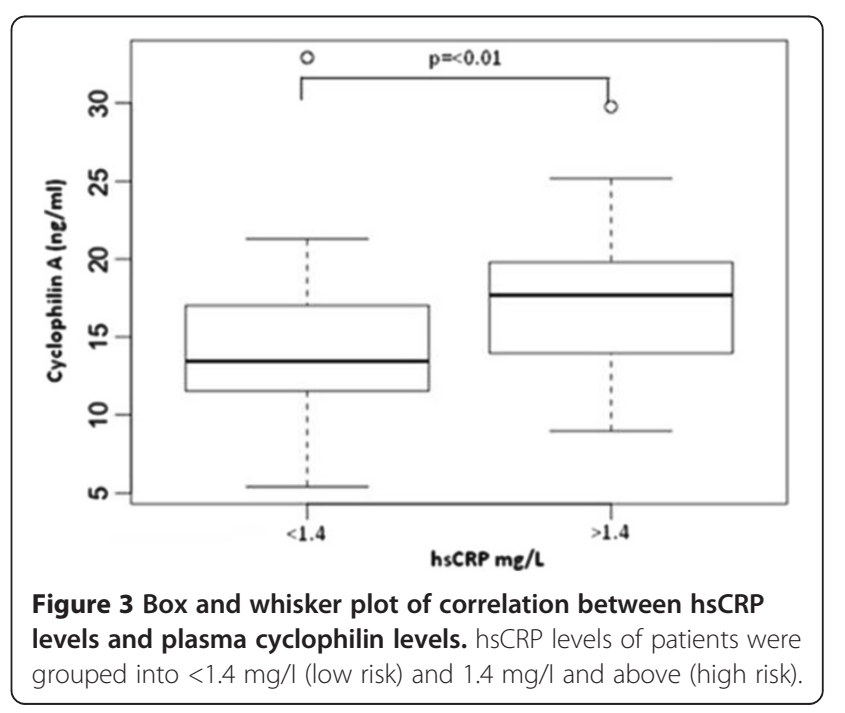


Table 4 Association of medications for diabetes, coronary artery disease and plasma levels of cyclophilin

\begin{tabular}{|c|c|c|c|c|c|c|c|}
\hline Medication* & Mean & Median & Std Dev & Min range & Max range & $\mathrm{T}$ value & $P$ value \\
\hline \multicolumn{8}{|c|}{ (i) Antiplatelets } \\
\hline Yes & 15.31 & 15.58 & 3.2 & 9.03 & 5.54 & 1.52 & 0.12 \\
\hline No & 14.67 & 15.12 & 3.6 & 5.54 & 19.93 & & \\
\hline \multicolumn{8}{|l|}{ (ii) Statins } \\
\hline Yes & 15.25 & 15.58 & 3.3 & 9.03 & 22.73 & 1.28 & 0.20 \\
\hline No & 14.71 & 15.38 & 3.5 & 5.54 & 19.93 & & \\
\hline \multicolumn{8}{|c|}{ (iii) Antihypertensive } \\
\hline Yes & 15.14 & 15.54 & 3.3 & 6.92 & 22.73 & 0.84 & 0.40 \\
\hline No & 14.78 & 15.4 & 3.5 & 5.54 & 19.93 & & \\
\hline \multicolumn{8}{|c|}{ (iv) Metformin } \\
\hline Yes & 15.75 & 16.58 & 3.2 & 10.28 & 22.73 & 4.00 & $<0.001$ \\
\hline No & 14.1 & 14.33 & 3.5 & 5.54 & 19.92 & & \\
\hline
\end{tabular}

*Medications were grouped into (i) antiplatelet aggregating agents (Ecospirin, Clopidogrel, Cilostazol) (ii) Statins (atorvastatin, lovastatin) (iii) Antihypertensive agents (Calcium channel blockers, ACE inhibitors, vasodilators and (iv) metformin derivatives (Glucophage, glycimet).

also higher in plasma of diabetes patients who also had coronary artery disease.

\section{Cyclophilin as an oxidative stress induced secretory factor} Cyclophilin A has been demonstrated to be an important secreted oxidative stress induced factor [6]. Cyclophilin A is secreted from vascular smooth muscle cells (VSMCs) [7] and endothelial cells (EC) [8] in response to reactive oxygen species (ROS). Cyclophilin A also stimulates smooth muscle cell migration and proliferation, endothelial cell adhesion molecule expression and chemotaxis of inflammatory cells. Studies on cyclophilin knockout mice models have demonstrated increased atherosclerotic potential clearly indicating its role in vascular disease progression [6]. Elevated levels of extracellular cyclophilin have also been detected in the synovial fluid of patients with rheumatoid arthritis [9]. Studies have reported an interaction between extracellular cyclophilin and CD 147 expressed by macrophages which may be responsible for development of arthritis [10]. Cyclophilin A has been shown to elicit inflammatory responses and thus contribute to recruitment of circulating blood cells during inflammatory response. Secreted cyclophilin A also contributes to maintaining blood brain barrier integrity and reducing tissue damage following brain injury. It reduces brain permeability by activating survival and growth pathways. Secreted cyclophilin A activates endothelial cells, a major component of blood brain barrier which in turns contributes to recruitment of circulatory monocytes to aid in blood brain barrier repair [11]. Given that cyclophilin A is a potent chemotactic agent for immune cells, particularly monocytes; it is plausible that this immunophilin plays an important role in accelerating atherosclerosis in type 2 diabetes.
Apart from its secretory features, cyclophilin has also gained recognition from a methodological point of view. As diabetes can modulate heart glyceraldehyde 3phosphate dehydrogenase (GAPDH) content expression, data can be normalized to cyclophilin expression [12]. Moreover, target gene expression may be calculated by using the expression of the cyclophilin housekeeping gene as an internal standard [13].

\section{Plasma cyclophilin A levels in diabetes and coronary artery disease}

We had earlier reported that high glucose induces secretion of cyclophilin A from monocytes [4]. We also observed a decrease in expression of this protein in the monocytes of patients with diabetes mellitus when compared to its expression in healthy controls [4]. Hyperglycaemia in diabetes and related oxidative stress could contribute to secretion of cyclophilin A from circulating monocytes and a rise in plasma cyclophilin A levels as seen in our patients with diabetes. Plasma cyclophilin levels have been assessed earlier in patients with coronary artery disease to study the severity of CAD using an immunoassay similar to the one we employed in our present study [14]. Satoh et al. divided cyclophilin values into equal quartiles for their analysis. A positive correlation was noted between plasma cyclophilin levels and coronary stenosis. Higher quartiles of plasma cyclophilin levels were associated with the need for coronary intervention. We also observed that patients with CAD, irrespective of whether they had diabetes had significantly higher cyclophilin A levels than those without CAD. This indicates the possible role of cyclophilin $\mathrm{A}$ in the pathogenesis of vascular disease. We had divided the patients into two groups based on when CAD was detected in them. This was done to evaluate whether cyclophilin 
A levels in plasma were different in chronic patients. Interestingly cyclophilin A levels were higher in chronic diabetes with CAD group. The significance of this finding needs to be evaluated through a prospective study.

The high prevalence of diabetes with and without coronary artery disease in those with high blood cyclophilin levels revealed in multinomial logistic regression analysis indicate a strong association of high plasma cyclophilin values with diabetes and vascular disease. The lower degree of association of high plasma cyclophilin levels with diabetes compared to those patients with only coronary artery disease could perhaps be explained by the possible higher mortality and lower survival among with those with multiple disease, resulting in lower prevalence.

Our study also demonstrates that classical risk factors and markers of diabetes, such as age, fasting blood sugar (FBS) and glycated hemoglobin (HbA1c) were positively associated with plasma cyclophilin levels indicating a specific relation of plasma cyclophilin levels with hyperglycemia. Other parameters such as gender, family history of diabetes, serum levels of high and low density lipoproteins and triglycerides were not associated with increase in cyclophilin levels. Satoh et al. also found that age, diabetes and hsCRP correlate with plasma cyclophilin levels in their patients with stenotic coronary arteries [14].

\section{Plasma cyclophilin $\mathrm{A}$ is associated with $\mathrm{C}$ reactive protein levels}

We next considered the correlation between plasma cyclophilin $\mathrm{A}$ and serum $\mathrm{C}$ reactive protein (CRP), a clinical marker of vascular inflammation [5]. CRP, an acute phase reactant has been developed as a surrogate marker of inflammatory mediators in coronary artery disease and diabetes [15-18]. The classical prospective study by Ridker et al. found elevated CRP to be an independent risk determinant of type 2 diabetes in healthy middle aged women [19]. CRP levels are observed to be higher in many diseases such as infections, trauma, or malignancy, as well as inflammatory, allergic, or necrotic diseases [20]. We evaluated the role of cyclophilin A among patients with high and low levels of serum CRP. We had looked into serum levels of CRP in a small limited subset of 77 individuals. Patients with low CRP $(<1 \mathrm{mg} / \mathrm{L})$ are at low risk and those with higher CRP $(>1 \mathrm{mg} / \mathrm{L})$ are at moderate or high risk for future cardiovascular events. We observed a positive correlation between plasma cyclophilin A and serum CRP levels. There was a positive association between patients with higher hsCRP levels and those with elevated plasma cyclophilin A in patients with diabetes as well as those with diabetes and CAD. Correlation (Pearson) Co-efficients of cyclophilin values were however, not significant between CRP and cyclophilin values possibly due to limited sample subset. A study by Satoh et al. [14] had suggested the use of cyclophilin A in conjunction with CRP as a predictor of risk in coronary artery disease in a study comprising of 320 patients with CAD. These findings suggest the possible utility of plasma cyclophilin A level as a marker of proinflammatory status in patients with diabetes.

\section{Metformin may reduce plasma cyclophilin levels}

To understand the variation in cyclophilin levels in relation to use of medication for diabetes and coronary artery disease, we looked into the association of plasma cyclophilin levels with the intake of medicines prescribed for these diseases. Cardiovascular drugs such as aspirin, clopidogrel and statins did not have any effect on plasma cyclophilin levels, whereas metformin intake was associated with decreased plasma cyclophilin levels. Metformin is a standard hypoglycemic drug used in treatment of non-insulin dependent diabetes mellitus [21]. The action of this drug is at the skeletal muscle, increasing glucose transport across the cell membrane [22]. Metformin is also known to reduce gene expression of inflammatory mediators such as IL-1 and Monocyte chemoattractant protein (MCP)-1 [23]. Metformin also reduces gene expression of an isoform of cyclophilin, cyclophilin D [24]. It is therefore, tempting to speculate that our observation of reduced plasma cyclophilin A in our patients taking metformin is related to metformin action on cyclophilin A gene expression in monocytes. This hypothesis however needs scrutiny.

In summary, our study reveals that patients with type 2 diabetes have higher circulating levels of the immunophilin cyclophilin A. Our observations that plasma cyclophilin $\mathrm{A}$ is higher in patients with type 2 diabetes irrespective of whether they have coronary artery disease or not indicates that hyperglycemia has an effect on cyclophilin secretion. Given that cyclophilin is known to be secreted from monocytes and vascular wall cells in conditions of oxidative stress such as hyperglycemia, the plasma circulating levels of cyclophilin A in patients with diabetes and CAD possibly reflects an increased oxidative stress and proinflammatory status in these conditions. Cyclophilin A thus needs to be evaluated as a marker of proinflammatory status in type 2 diabetes and possibly a predictor of early vascular disease through a prospective study in a large population of diabetic subjects.

\section{Abbreviations}

ANOVA: Analysis of Variance; CAD: Coronary artery disease; CRP: C reactive protein; DM: Diabetes Mellitus; EDTA: Ethylene diamine tetra cetic acid; ELISA: enzyme-linked immunosorbent assay; FBS: fasting blood sugar; GAPDH: Glyceraldehyde 3-phosphate dehydrogenase; HbA1c: Glycated haemoglobin; HDL: High density lipoprotein; LDL: Low density lipoprotein; VSMCs: Vascular smooth muscle cells.

\section{Competing interests}

All authors declare that they have no competing interests. 


\section{Authors' contributions}

SR and CCK designed the study and wrote the paper; AV, VA and DG carried out the experiments; VR, VC, AM, NSP, KRS and MRP planned, executed the study and helped in analyzing the data. All authors read and approved the final manuscript.

\section{Acknowledgements}

This work was supported by Indian Council of Medical Research, Ministry of Health, Government of India.

\section{Author details}

${ }^{1}$ Cardiovascular Disease Biology, Rajiv Gandhi Centre for Biotechnology, Thiruvananthapuram, India. ${ }^{2}$ Achutha Menon Centre, SCTIMST, Thiruvananthapuram, India. ${ }^{3}$ Madras Medical Mission, Chennai, India. ${ }^{4}$ Indian Institute of Diabetes, Thiruvananthapuram, India. ${ }^{5}$ PRS Hospital,

Thiruvananthapuram, India. ${ }^{6}$ Cancer Research Program, Rajiv Gandhi Centre for Biotechnology, Thiruvananthapuram, India.

Received: 12 December 2013 Accepted: 5 February 2014 Published: 7 February 2014

\section{References}

1. Satoh K, Shimokawa H, Berk BC, Cyclophilin A: Promising new target in cardiovascular therapy. Circ J 2010, 74:2249-2256.

2. Jin ZG, Melaragno MG, Liao DF, Yan C, Haendeler J, Suh YA, Lambeth JD, Berk BC: Cyclophilin A is a secreted growth factor induced by oxidative stress. Circ Res 2000, 87:789-796.

3. Satoh K, Nigro P, Berk BC: Oxidative stress and vascular smooth muscle cell growth: a mechanistic linkage by cyclophilin A. Antioxid Redox Signal 2010, 12:675-682.

4. Ramachandran S, Venugopal A, Sathisha K, Reshmi G, Charles S, Divya G, et al: Proteomic profiling of high glucose primed monocytes identifies cyclophilin A as a potential secretory marker of inflammation in type 2 diabetes. Proteomics 2012, 12:2808-2821.

5. Ridker PM: C-reactive protein: a simple test to help predict risk of heart attack and stroke. Circulation 2003, 108:81-85.

6. Nigro P, Satoh K, O'Dell MR, Soe NN, Cui Z, Mohan A, Abe J, Alexis JD, Sparks JD, Berk BC: Cyclophilin A is an inflammatory mediator that promotes atherosclerosis in apolipoprotein E-deficient mice. J Exp Med 2011, 208:53-66.

7. Jin ZG, Melaragno MG, Liao DF, Yan C, Haendeler J, Suh YA, Lambeth JD, Berk BC: Cyclophilin A is a secreted growth factor induced by oxidative stress. Circ Res 2000, 87:789-796.

8. Zheng-Gen J, Andreea Lungu O, Liang X, Meng W, Chelsea W, Bradford C: BerkCyclophilin A Is a Proinflammatory Cytokine that Activates Endothelial Cells. Arterioscler Thromb Vasc Biol 2004, 24:1186-1191.

9. Yurchenko V, Constant S, Bukrinsky M: Dealing with the family: CD147 interactions with cyclophilins. Immunology 2006, 117:301-309.

10. Yang Y, Lu N, Zhou J, Chen Z n, Zhu P: Cyclophilin A up-regulates MMP-9 expression and adhesion of monocytes/macrophages via CD147 signalling pathway in rheumatoid arthritis. Rheumatology 2008, 47:1299-1310.

11. Redell JB, Zhao J, Dash PK: Acutely increased cyclophilin a expression after brain injury: A role in blood-brain barrier function and tissue preservation. J Neurosci Res 2007, 85:1980-1988.

12. Rosa CM, Xavier NP, Henrique Campos D, Fernandes AA, Cezar MD, Martinez PF, Cicogna AC, Gimenes C, Gimenes R, Okoshi MP, Okoshi K: Diabetes mellitus activates fetal gene program and intensifies cardiac remodeling and oxidative stress in aged spontaneously hypertensive rats. Cardiovasc Diabetol 2013, 12:152.

13. Atalar F, Gormez S, Caynak B, Akan G, Tanriverdi G, Bilgic-Gazioglu S, Gunay D, Duran C, Akpinar B, Ozbek U, Buyukdevrim AS, Yazici Z: The role of mediastinal adipose tissue 11-hydroxysteroid dehydrogenase type 1 and glucocorticoid expression in the development of coronary atherosclerosis in obese patients with ischemic heart disease. CardiovascDiabetol 2012, 11:115.

14. Satoh K, Fukumoto $Y$, Sugimura $K$, et al: Plasma cyclophilin $A$ is a novel biomarker for coronary artery disease. Circ J 2013, 77:447-455.

15. Koenig W, Sund M, Frohlich $M$, et al: C-reactive protein, a sensitive marker of inflammation, predicts future risk of coronary heart disease in initially healthy middle-aged men: results from the MONICA (Monitoring Trends and Determinants in Cardiovascular Disease) Augsburg Cohort Study, 1984 to 1992. Circulation 1999, 99:237-242.

16. Tracy RP, Lemaitre RN, Psaty BM, et al: Relationship of C-reactive protein to risk of cardiovascular disease in the elderly: results from the Cardiovascular Health Study and the Rural Health Promotion Project. ArteriosclerThrombVasC Biol 1997, 17:1121-1127.

17. Pradhan AD, Manson JE, Rifai N, et al: C-reactive protein, interleukin 6, and risk of developing type 2 diabetes mellitus. JAMA 2001, 286:327-334.

18. Pepys MB: C-reactive protein fifty years on. Lancet 1981, 1:653-657.

19. Ridker PM, Buring JE, Shih J, et al: Prospective study of C-reactive protein and the risk of future cardiovascular events among apparently healthy women. Circulation 1998, 98:731-733.

20. Blankenberg S, Yusuf S: The inflammatory hypothesis: any progress in risk stratification and therapeutic targets. Circulation 2006, 114:1557-1560.

21. Klip A, Leiter LA: Cellular mechanism of action of metformin. Diabetes Care 1990, 13:696-704.

22. Cusi K, Consoli A, DeFronzo RA: Metabolic effects of metformin on glucose and lactate metabolism in noninsulin-dependent diabetes mellitus. J ClinEndocrino/Metab 1996, 81:4059-4067.

23. Moreno-Navarrete JM, Ortega FJ, Rodríguez-Hermosa Jl, Sabater M, Pardo G, Ricart W, Fernández-Real JM: OCT1 expression in adipocytes could contribute to increased metformin action in obese subjects. Diabetes 2011, 60:168-176.

24. Lablanche S, Cottet-Rousselle C, Lamarche F, Benhamou PY, Halimi S, Leverve XE: Fontaine Protection of pancreatic INS-1 beta-cells from glucose- and fructose-induced cell death by inhibiting mitochondrial permeability transition with cyclosporin A or metformin. Cell Death Dis 2011, 2:134

doi:10.1186/1475-2840-13-38

Cite this article as: Ramachandran et al:: Plasma level of cyclophilin A is increased in patients with type 2 diabetes mellitus and suggests presence of vascular disease. Cardiovascular Diabetology 2014 13:38.

\section{Submit your next manuscript to BioMed Central and take full advantage of:}

- Convenient online submission

- Thorough peer review

- No space constraints or color figure charges

- Immediate publication on acceptance

- Inclusion in PubMed, CAS, Scopus and Google Scholar

- Research which is freely available for redistribution 